\title{
Role of computed tomographic scanning of the thorax prior to bronchoscopy in the investigation of suspected lung cancer
}

Clare Laroche, Ian Fairbairn, Hilary Moss, Joanna Pepke-Zaba, Linda Sharples, Chris Flower, Richard Coulden

\begin{abstract}
Background-Fibreoptic bronchoscopy (FOB) is the usual initial investigation of choice in patients with suspected endobronchial carcinoma, but it is often nondiagnostic. Once a positive diagnosis has been made, many patients undergo staging by computed tomographic (CT) scanning to assess the extent of the disease and its suitability for radical treatment. To determine whether initial CT scanning before FOB is a cost effective way of reducing subsequent unnecessary or unhelpful invasive diagnostic procedures, a study was undertaken in 171 patients with suspected endobronchial carcinoma.

Methods-A randomised two group study was performed with all patients undergoing an initial CT staging scan. In group $A$ the CT scans were reviewed before FOB, allowing cancellation or a change to an alternative invasive procedure if considered appropriate. In group $B$ all patients proceeded to FOB with the bronchoscopist blinded to the result of the CT scan until after the procedure.
\end{abstract}

Results-In group A six of 90 patients (7\%) required no further investigations as the CT scan was either normal, consistent with benign disease, or consistent with widespread metastatic disease. Of the remainder, bronchoscopy was diagnostic in 50 of $68(73 \%)$ in group A compared with 44 of $81(54 \%)$ in group $B(p=0.015)$. Overall, a positive diagnosis was made after a single invasive investigation in 64 of 84 patients $(76 \%)$ in group A compared with only 45 of 81 patients $(55 \%)$ in group $B(p=0.005)$. Only seven of 90 patients $(8 \%)$ in group $A$ required more than one invasive investigation compared with 15 of 81 patients $(18.5 \%)$ in group $B$. In patients with malignancy, bronchoscopy was more likely to be diagnostic in group A (50 of 56 patients $(89 \%)$ ) than in group B (44 of 62 $(71 \%) ; p=0.012)$, and the diagnosis was more frequently made on the initial invasive investigation (group A, 63 of 70 (90\%); group $B, 44$ of $62(71 \%) ; p=0.004)$. Because of the lower number of invasive procedures performed in group $A$ than in group $B$, the cost of performing CT scans before FOB in all patients in group $A$ would have equated to a projected cost of performing CT scans in $60 \%$ of patients after FOB in group B.
Conclusions-Performing initial CT thoracic scans before bronchoscopy in patients with suspected endobronchial malignancy is a cost effective way of improving diagnostic yield from invasive diagnostic procedures and occasionally may obviate the need for any further investigation.

(Thorax 2000;55:359-363)

Keywords: lung cancer; diagnosis; computed tomography; fibreoptic bronchoscopy

Most patients who present with chest radiographic appearances suggestive of bronchial carcinoma undergo fibreoptic bronchoscopy (FOB) as the primary investigation of choice. However, in a significant proportion of patients bronchoscopy is non-diagnostic because the lesion is outside the bronchus or in the periphery of the lung. In some centres patients with a peripheral mass on the chest radiograph may undergo a percutaneous needle biopsy as the initial investigation, although the expertise in this procedure is not as widespread as for bronchoscopy. It is also associated with a higher complication rate.

Computed tomographic (CT) scanning of the thorax and upper abdomen has a well recognised role in the staging of bronchial carcinoma prior to definitive treatment, once a tissue diagnosis has been obtained..$^{1-4}$ The potential benefits of performing CT scans in all patients with suspected endobronchial carcinoma prior to FOB have not previously been explored. These could include a more appropriate choice of initial investigation or, in some cases, elimination of the need for invasive investigation altogether. When bronchoscopy is performed, knowledge of the CT appearance could improve the diagnostic yield by better targeting of suspicious lesions. This applies to both endobronchial disease visible at FOB and transbronchial biopsy specimens and washings taken from the periphery of the lung. ${ }^{56}$ Transbronchial needle aspiration and biopsy results are also enhanced by knowledge of the appearance of the CT scan. ${ }^{7-9}$

A randomised prospective study, designed to assess the benefit of performing CT scans as the primary procedure in all patients presenting to a lung cancer investigation unit with suspected endobronchial carcinoma, is described.

\section{Methods}

Our pulmonary oncology service organises the investigations of all new outpatient referrals 
and most inpatient referrals with suspected lung cancer from three surrounding health districts. ${ }^{10}$ Only about $10 \%$ of patients in these health districts are diagnosed outside the service, usually because they are too unwell to travel or because the diagnosis is not suspected at the time of bronchoscopy; these patients, and those from a fourth health district which was only referring patients for needle biopsies at the time of the study were not included in the study.

Patients were seen initially at their local hospital where a full history was obtained and a clinical examination and chest radiography performed. The patient was informed that a diagnosis of lung cancer was suspected and that they would be referred to Papworth Hospital for further investigation and management. The patient's notes and chest radiograph were then reviewed in the oncology centre by a chest physician and radiologist. All patients referred in this way were considered for inclusion in the study. Only patients in whom bronchoscopy was considered the most likely route of obtaining a positive tissue diagnosis (chest radiographic appearances of a proximal tumour with or without distal collapse or consolidation, or diffuse shadowing considered amenable to transbronchial biopsy; and/or patients with haemoptysis, recent onset of a new cough, fixed wheeze or stridor) were randomised into the study. Patients in whom bronchoscopy was considered unlikely to be diagnostic (absence of suggestive clinical signs or symptoms, presence of a clearly defined peripheral mass on chest radiograph, suitable for needle biopsy, with no proximal component) were excluded since needle biopsy is readily available in our institution and is associated with a high positive diagnostic rate.

One hundred and seventy two consecutive patients selected for bronchoscopy from June 1996 onwards were invited to take part in the study. Informed consent was sought by the radiologist before a CT scan was performed. Only one patient refused consent and the remaining 171 patients were randomised to two groups according to a computer generated simple randomisation list. Group allocations were held in opaque sealed envelopes and opened after signed informed consent was obtained.

All patients underwent a staging CT scan as the initial investigation on the assessment day. CT scanning was performed using a modern spiral scanner (Somatom Plus 4a, Siemens, Erlangen, Germany). Spiral scans of the whole thorax were obtained with $10 \mathrm{~mm}$ collimation and these were followed by a further targeted spiral sequence during intravenous contrast enhancement with $3 \mathrm{~mm}$ collimation. Postcontrast images of the liver and adrenal glands were then obtained.

For patients in group A the CT findings were immediately discussed with the bronchoscopist before any further procedure took place. Patients either then proceeded to bronchoscopy, underwent an alternative procedure or, in a few cases, required no further investigations. All patients in group B underwent immediate bronchoscopy, with the bronchoscopist blinded to the results of the CT examination until after bronchoscopy had been performed. In both groups at least five bronchial biopsy specimens were taken of visible tumours, and bronchial washings were performed in all patients; bronchial brushings were taken at the discretion of the bronchoscopist if there was concern about the quality of the bronchial biopsy specimens. All patients in whom no tumour was visible underwent bronchial washings; biopsy specimens and brushings were taken if any mucosal abnormalities were identified. If the initial investigation was non-diagnostic, a second diagnostic investigation (such as percutaneous needle biopsy) was performed at a later date, if judged appropriate, after discussion between the bronchoscopist and radiologist with reference to the $\mathrm{CT}$ appearances.

\section{DATA ANALYSIS}

Data were collected on the type of initial invasive investigation, its diagnostic yield, and complication rate. Comparability of the two groups was assessed using the Student's unpaired $t$ test for age, Pearson's $\chi^{2}$ test for contingency tables for type of malignancy, and Fisher's exact test for diagnosis at first investigation and incidence of complications. Exact two sided significance levels are presented. The cost effectiveness of performing an initial CT examination was assessed by comparing the average cost of obtaining a positive tissue diagnosis in group A with that in group B, assuming a projected CT scan rate of $60 \%$ in group B.

\section{Results}

One hundred and seventy one patients were randomised into the study (90 in group A and 81 in group B). The groups were comparable in age (mean 67.0 years (range $41-85$ ) in group A, mean 67.8 years (range 38-88) in group B) and sex distribution $(62 \%$ men in both groups). Overall, 134 patients (78\%) were diagnosed as having a malignancy (72 of 90 $(80 \%)$ in group $\mathrm{A} ; 62$ of $81(76.5 \%)$ in group B) and there was no significant difference between the groups in the type of malignant disease (table $1, \mathrm{p}=0.389$ ).

In group A 68 of 90 patients $(76 \%)$ underwent bronchoscopy as their initial investigation. The CT appearances suggested that an alternative investigation would be more appropriate in 16 patients (figs 1 and 2). Six patients in group A had no invasive investigations: two with presumed malignancy (one had

Table 1 Final diagnosis

\begin{tabular}{lcc}
\hline & $\begin{array}{c}\text { Group } A \\
(n=90)\end{array}$ & $\begin{array}{l}\text { Group B } \\
(n=81)\end{array}$ \\
\hline Bronchial carcinoma & $28(31.1 \%)$ & $24(29.6 \%)$ \\
Squamous cell carcinoma & $9(10.0 \%)$ & $13(16.0 \%)$ \\
Adenocarcinoma & $9(10.0 \%)$ & $7(8.6 \%)$ \\
Small cell carcinoma & $15(16.7 \%)$ & $5(6.2 \%)$ \\
Large cell carcinoma & $2(2.2 \%)$ & $4(4.9 \%)$ \\
Mixed & $9(10.0 \%)$ & $9(11.1 \%)$ \\
Other malignant disease & $18(20.0 \%)$ & $19(23.5 \%)$ \\
Non-malignant disease & & \\
\hline
\end{tabular}




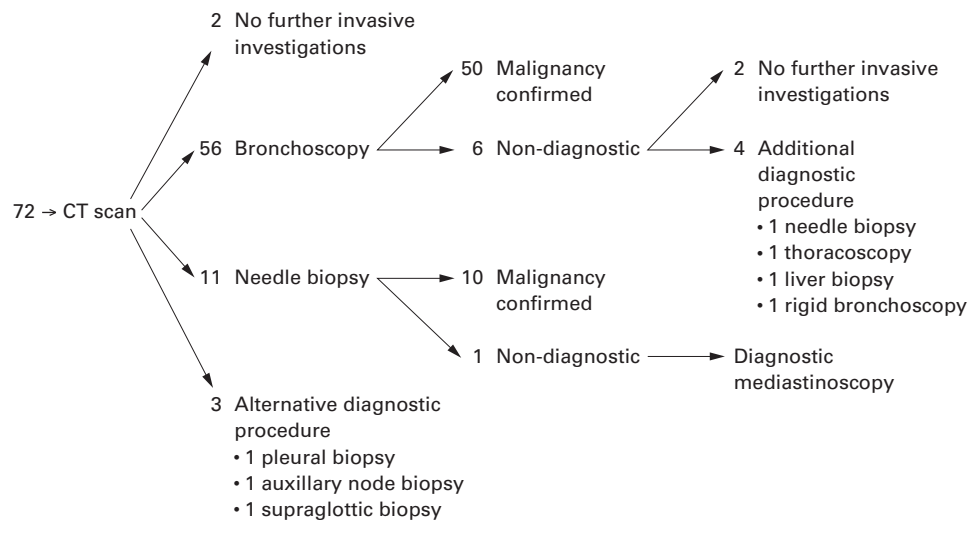

Figure 1 Group A:patients with malignancy.

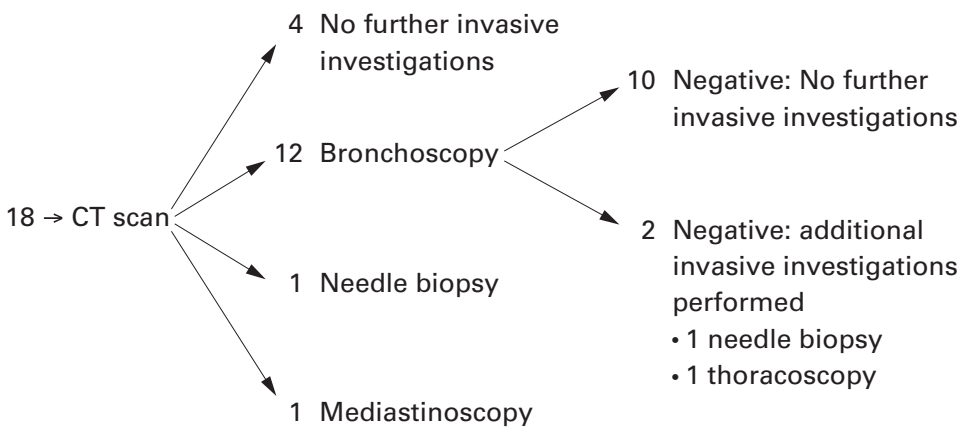

Figure 2 Group A:patients with no malignancy.

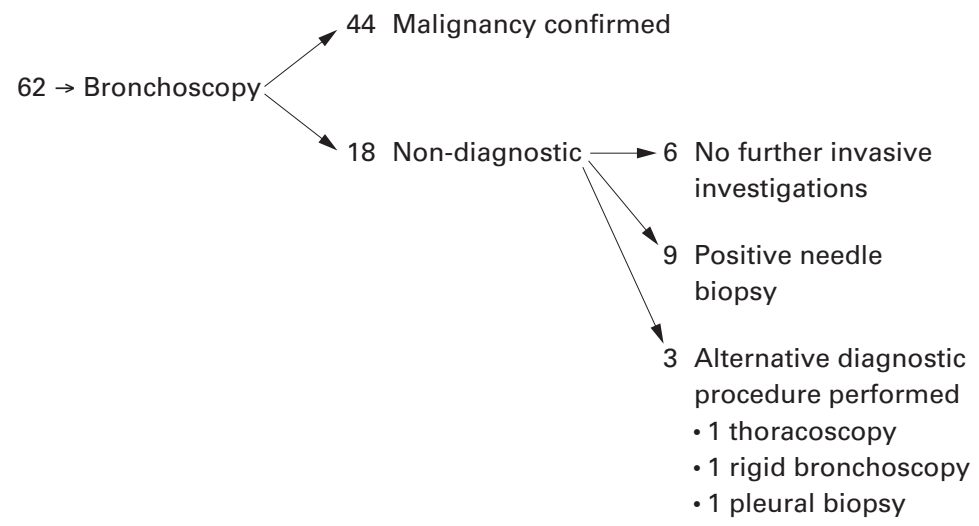

Figure 3 Group B: patients with malignancy.

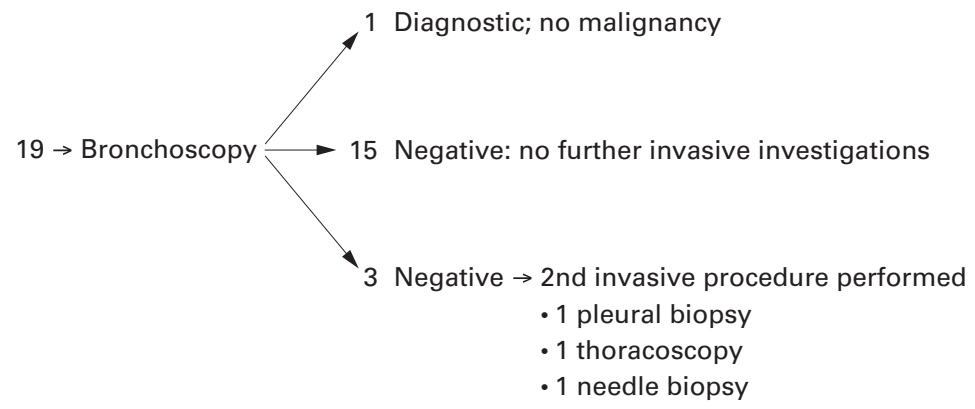

Figure 4 Group B:patients with no malignancy.

a positive sputum and one had widespread cancer) and in four patients the CT scan was either normal or showed benign disease (such as bronchiectasis).

Of the 68 patients in group A who underwent bronchoscopy as their initial diagnostic procedure, a tissue diagnosis was obtained in $50(73 \%)$ compared with 44 of 81 patients (54\%) in group B (figs 3 and 4). Overall, a diagnosis was made from the initial invasive investigation in 64 of 84 patients $(76 \%)$ in group A and 45 of 81 (55\%) in group B (table $2, \mathrm{p}=0.005)$. All patients in group $\mathrm{B}$ underwent bronchoscopy in accordance with the agreed protocol.

In patients with confirmed malignancy, bronchoscopy was diagnostic in 50 of 56 patients $(89 \%)$ in group A (fig 1) compared with only 44 of $62(71 \%)$ in group B (fig 3, table $3, p=0.012)$. There was also a significant increase in the number of patients with malignant disease receiving a histological diagnosis on initial invasive investigation (63 of $70(90 \%)$ in group $\mathrm{A}$ and 44 of $62(71 \%)$ in group B; $\mathrm{p}=$ $0.004)$.

Of the 50 diagnostic bronchoscopies in patients with malignancy in group A, 46 patients were diagnosed by positive biopsy specimens of a visible tumour (31 also had positive washings, 12 also had positive brushings), one patient was diagnosed by a positive brushing alone of a visible tumour, and three patients were diagnosed on the basis of a "blind" washing. In the six patients in whom bronchoscopy was non-diagnostic, tumour was seen in two patients, biopsy specimens were taken in five, brushings in two, and washings in all six.

Of the 44 diagnostic bronchoscopies in patients with malignancy in group $\mathrm{B}, 38$ patients were diagnosed by positive biopsy specimens of a visible tumour (28 also had positive washings, seven also had positive brushings), two were diagnosed by a positive washing of a visible tumour (one also had a positive brushing), and four were diagnosed on the basis of a "blind" washing (one of these also had a positive "blind" brushing). In the 18 patients with a negative bronchoscopic examination tumour was visible in four patients, biopsies were attempted in 15 , brushings in nine, and washings in all 18 patients.

Overall, the improved diagnostic accuracy of bronchoscopy in group A appeared to relate primarily to a higher proportion of visible tumours, which is to be expected since patients in group A with no endobronchial component to their tumour on CT scanning would have had an alternative procedure. However, there was a higher rate of non-diagnosis when tumour was visible in group B (four of 44, 9\%) than in group A (two of $49,4 \%$ ). In patients with no visible tumour there was no difference between the proportion of patients undergoing bronchial biopsies (five of seven in group A; 14 of 18 in group B) or bronchial brushings (three of seven in group A; 10 of 18 in group B).

Patients in whom malignancy was not found have been followed up for a period of at least 24 months and, as yet, no new evidence of malignancy has emerged.

Complications were rare and the rates were comparable in the two groups (table 4). There were no deaths or major haemorrhages secondary to the investigations in either group. A minor pneumothorax (classified as one that was asymptomatic, less than $30 \%$, and did not 
Table 2 Success of initial invasive investigation in all patients

\begin{tabular}{lcll}
\hline & $\begin{array}{l}\text { Group } A \\
(n=90)\end{array}$ & $\begin{array}{l}\text { Group B } \\
(n=81)\end{array}$ & p value \\
\hline No invasive investigations & $6 / 90(7 \%)$ & N/A & \\
Diagnostic FOB & $50 / 68(73 \%)$ & $44 / 81(54 \%)$ & 0.015 \\
Diagnostic CT biopsy & $10 / 12(83 \%)$ & N/A & \\
Other diagnostic investigation & $4 / 4(100 \%)$ & N/A & \\
Diagnosis made on initial invasive investigation & $64 / 84(76 \%)$ & $45 / 81(55 \%)$ & 0.005 \\
\hline
\end{tabular}

Table 3 Success of initial investigation in patients with confirmed malignancy

\begin{tabular}{lcll}
\hline & Group A & Group B & p value \\
\hline Total number of patients & $72(80 \%)$ & $62(76 \%)$ & \\
No invasive investigations & $2 / 72(3 \%)$ & $\mathrm{N} / \mathrm{A}$ & \\
Diagnostic bronchoscopy & $50 / 56(89 \%)$ & $44 / 62(71 \%)$ & 0.012 \\
Diagnostic CT biopsy & $10 / 11(91 \%)$ & $\mathrm{N} / \mathrm{A}$ & \\
Other diagnostic investigation & $3 / 3(20 \%)$ & $\mathrm{N} / \mathrm{A}$ & \\
Histological diagnosis made on initial investigation & $63 / 70(90 \%)$ & $44 / 62(71 \%)$ & \multirow{2}{*}{0.004} \\
\hline
\end{tabular}

Table 4 Number of invasive investigations and complications: all patients

\begin{tabular}{lccc}
\hline & $\begin{array}{c}\text { Group } A \\
(n=90)\end{array}$ & $\begin{array}{c}\text { Group } B \\
(n=81)\end{array}$ & p value \\
\hline CT alone & $6(7 \%)$ & 0 & \\
One invasive investigation & $77(85 \%)$ & $66(81.5 \%)$ & \\
Two invasive investigations & $7(8 \%)$ & $15(18.5 \%)$ & \\
Major complications & 0 & $1(1.2 \%)$ & \\
Pneumothorax requiring conservative treatment & $3(3.3 \%)$ & $4(4.9 \%)$ & 0.247 \\
Minor haemorrhage & $2(2.2 \%)$ & $1(1.2 \%)$ & 0.262 \\
\hline
\end{tabular}

Table 5 Total cost of diagnostic investigations for each group based on a CT scan rate before fibreoptic bronchoscopy (FOB) in group $A$ of $100 \%$ and a projected CT scan rate after bronchoscopy in group $B$ of $60 \%$

\begin{tabular}{|c|c|c|c|c|c|}
\hline Tests & $\begin{array}{l}\text { Group A } \\
(n=90)\end{array}$ & $\begin{array}{l}\text { Group B } \\
(n=81)\end{array}$ & $\begin{array}{l}\text { Cost } \\
(\mathcal{E})\end{array}$ & $\begin{array}{l}\text { Total cost } \\
(\text { group A) }\end{array}$ & $\begin{array}{l}\text { Total cost } \\
\text { (group B) }\end{array}$ \\
\hline FOB & 68 & 81 & 387 & 26316 & 31347 \\
\hline CT thorax & 90 & 48 & 121 & 10890 & 5808 \\
\hline CT guided biopsy & 14 & 10 & 297 & 4158 & 2970 \\
\hline Mediastinoscopy & 2 & 0 & 1009 & 2018 & \\
\hline Rigid bronchoscopy & 1 & 1 & 1009 & 1009 & 1009 \\
\hline Liver biopsy & 1 & 0 & 53 & 53 & \\
\hline Thoracoscopic biopsy & 2 & 2 & 1009 & 2018 & 2018 \\
\hline Pleural biopsy & 1 & 2 & 53 & 53 & 106 \\
\hline Supraglottic biopsy & 1 & 0 & 459 & 459 & - \\
\hline Axillary node biopsy & 1 & 0 & 260 & 260 & \\
\hline Total cost $(£)$ & & & & 47234 & 43258 \\
\hline Cost per patient $(f)$ & & & & 525 & 534 \\
\hline
\end{tabular}

require any specific treatment) occurred in three patients in group A and four in group B. A major pneumothorax requiring intercostal tube drainage occurred in one patient in group B.

Table 5 shows the average cost of investigations at Papworth for patients in group A, with universal CT scanning before bronchoscopy, compared with an estimated cost for patients in group B assuming a projected CT rate after bronchoscopy of $60 \%$. The costs do not take into account overnight stays. At Papworth hospital both FOB and percutaneous biopsies are performed on a day care basis provided the patient is neither frail nor returning to an empty home.

\section{Discussion}

There have been a number of studies correlating CT scanning with bronchoscopic findings. In 1985 Colice et al reported that CT scans were moderately accurate in predicting airway abnormalities using $10 \mathrm{~mm}$ collimation (sensitivity $63-85 \%$; specificity $61-77 \%),{ }^{11}$ but they were inaccurate in defining the type of mucosal abnormality as they could not distinguish between localised mucosal abnormalities, endobronchial mass, or extrinsic compression. Similar conclusions were reached by Naidich et $a .^{6}$

Improved accuracy was reported by Mayr et al using $5 \mathrm{~mm}$ collimation. ${ }^{12}$ Their study of 142 patients with suspected endobronchial tumour showed sensitivities of $91-94 \%$ and a specificity of $99 \%$ in the diagnosis of a normal or narrowed bronchial lumen. They recommended CT scanning prior to bronchoscopy in patients with suspected carcinoma on the chest radiograph to guide the bronchoscopist and in those with a normal chest radiograph and normal or equivocal FOB if a bronchial tumour was still clinically suspected. With diagnostic performance improved by thin slice collimation (2$5 \mathrm{~mm}$ ), the main advantage of spiral CT scanning is improved delineation of the extent of the tumour and its relationship to adjacent structures, together with enhanced detection of minor bronchial abnormalities. Further advantages of spiral CT scanning include multiplanar reformatting of volume data sets and three dimensional surface displays which are complementary to bronchoscopy..$^{13}$ Our CT protocol also allowed formal staging of the tumour.

Despite the advantages of modern scanning techniques, FOB is required to detect subtle mucosal abnormalities and to obtain a tissue diagnosis. For these reasons it remains the investigation of choice for the inspection of major and segmental bronchi. ${ }^{14}$

The role of CT scanning in the investigation of haemoptysis has also been assessed in a number of studies. ${ }^{15-17}$ In this particular clinical setting CT scanning improves the diagnostic yield from bronchoscopy and should be the primary imaging investigation.

Our study is unique in that it is the only prospective investigation of the use of CT scanning prior to FOB in all patients with suspected endobronchial carcinoma and of its impact on management. Our results show that CT scanning before bronchoscopy benefits patients in a number of ways. A small number ( $7 \%$ of our patients) avoided the need for any invasive investigations as the CT scan was either normal or considered diagnostic. When bronchoscopy was performed a diagnosis was more likely when the bronchoscopist had the benefit of additional information from the CT scan on the site and characteristics of the presumptive tumour, allowing better targeting of the lesion. In patients without malignancy a CT scan allowed better targeting of abnormal areas. Finally, the number of investigations and days spent in hospital were reduced in patients undergoing CT scanning prior to bronchoscopy.

For ethical reasons the results of the CT scans in group B were reviewed immediately after bronchoscopy in all patients, thereby identifying the most appropriate second invasive investigation in patients with a nondiagnostic examination. This obviously contributed to the high histological confirmation rate in both groups, and is also likely to have reduced the apparent cost benefit of 
performing CT scans routinely before FOB (which is based on a projected CT scan rate of only $60 \%$ in group B).

During the period of our study we were not using transbronchial (Wang) needle aspiration routinely, but since this study we have begun using the technique to sample subcarinal nodes and/or extrabronchial masses at the time of the initial diagnostic bronchoscopy, and this is likely to increase further the cost effectiveness of CT scanning prior to bronchoscopy.

In a significant number of patients the CT appearances indicated either that there was unlikely to be an endobronchial component to a tumour and/or that a different invasive procedure (such as percutaneous biopsy) was more likely to be diagnostic than FOB, so reducing the proportion of patients at bronchoscopy without a visible tumour. In our study we attempted to exclude patients with an obvious peripheral mass amenable to percutaneous needle biopsy. In institutions where needle biopsy is less freely available, a higher proportion of patients are likely to undergo a bronchoscopic examination as their initial invasive investigation, and the benefit of prior CT scanning is therefore likely to be higher in this situation.

Although the theoretical risk of pneumothorax is greater in patients who undergo percutaneous biopsy than FOB biopsy, the complication rates were comparable in our two groups of patients. However, to detect a significant difference in complication rates similar to those shown in table 4 we would have needed to study 5550 patients.

The cost benefit analysis of performing CT scanning before FOB in all patients with suspected endobronchial carcinoma must allow for the fact that many patients with lung cancer undergo a CT examination after histological diagnosis to stage their disease, although the proportion of patients proceeding to a staging CT scan varies from centre to centre. The average cost of obtaining a histological diagnosis in group A was $£ 525$ per patient. Such a cost would equate to that of a projected cost of performing CT scans after bronchoscopy in approximately $60 \%$ of patients (table $5)$. In centres where CT scans are performed in about $60 \%$ of patients with proven or suspected lung cancer, it would appear to be cost effective to change to universal CT scanning before bronchoscopy since this significantly improves the diagnostic accuracy of bronchoscopy, allows about $7 \%$ of patients to avoid an unnecessary invasive procedure, and a further $10 \%$ to avoid the requirement for a second invasive investigation. In units where the named CT rate is higher or lower than $60 \%$, cost effectiveness would be correspondingly higher or lower. Similarly, where the CT scan was used to guide additional staging techniques such as Wang needle aspiration of enlarged lymph glands, cost effectiveness would be increased further.

Our oncology service is characterised by a desire to provide a histological diagnosis and subsequent treatment plan for patients with suspected lung cancer in the shortest possible time and with the least inconvenience to patients. CT scanning before bronchoscopy in patients with suspected endobronchial carcinoma improves the diagnostic accuracy of the first invasive test with little increase in cost and no change in patient risk. While this approach may not be transferable to all other units, in units such as ours, where an average of 8-10 patients with suspected lung cancer are investigated each week, the benefits of running a concurrent CT scan/needle biopsy/bronchoscopy list are considerable, allowing flexibility and developing the multidisciplinary approach to lung cancer.

We would like to thank Dr Miriam Scadang, Dr Susan Stewart, and Mrs Leigh Clements for their help in performing the study.

Funding: No external funding.

1 McLoud TC, Bourgouin PM, Greenberg RW, et al. Bronchogenic carcinoma: analysis of staging in the mediastinum with CT by correlative lymph node mapping and sampling. Radiology 1992;182:319-23.

2 Pearson FG. Staging of the mediastinum. Role of mediastinoscopy and computed tomography. Chest 1993;103:3468S.

3 Primack SL, Lee KS, Logan PM, et al. Bronchogenic carcinoma: utility of CT in the evaluation of patients with carcinoma: utility of CT in the evaluation of patien

4 Staples CA, Muller NL, Miller RR, et al. Mediastinal nodes in bronchogenic carcinoma: comparison between CT and mediastinoscopy. Radiology 1988;167:367-72.

5 Henschke CI, Davis SD, Auh Y, et al. Detection of bronchial abnormalities: comparison of CT and bronchoscopy. $\mathcal{F}$ Comput Assist Tomogr 1987;11:432-5.

6 Naidich DP, Lee JJ, Garay SM, et al. Comparison of CT and fibreoptic bronchoscopy in the evaluation of bronchial disease. AfR 1987;148:1-7.

7 Wang KP, Haponik EF, Britt EJ, et al. Transbronchial needle aspiration of peripheral pulmonary nodules. Chest $1984 ; 86$ : 819-23.

8 Naidich DP, Sussman R, Kutcher WL, et al. Solitary pulmonary nodules, CT-bronchoscopic correlation. Chest 1988; 93:595-8.

9 Gaeta M, Barone M, Russi EG, et al. Carcinomatous solitary pulmonary nodules: evaluation of the tumourbronchi relationship with thin-section CT. Radiology 1993; 187:535-9.

10 Laroche CM, Wells F, Coulden RC, et al. Improving surgical resection rate in lung cancer. Thorax 1998;53:445-9.

11 Colice GL, Chappel GJ, Frenchman SM, et al. Comparison of computerised tomography with fibreoptic bronchoscopy in identifying endobronchial abnormalities in patients with known or suspected lung cancer. Am Rev Respir Dis 1985; 131:397-400.

12 Mayr B, Ingrisch $\mathrm{H}$, Haussinger $\mathrm{K}$, et al. Tumors of the bronchi: role of evaluation with CT. Radiology 1989;172: 647-52.

13 Fleiter T, Merkle EM, Aschoff AJ, et al. Comparison of realtime virtual and fibreoptic bronchoscopy in patients with bronchial carcinoma: opportunities and limitations. AfR 1997;169:1591-5

14 Chinn RJS, Yang GZ, Congleton J, et al. Three-dimensional computed tomography bronchoscopy using clinical datasets: a comparison with fibreoptic bronchoscopy. Clin datasets: a comparison
Radiol 1997;52:830-6.

15 Set PAK, Flower CDR, Smith IE, et al. Hemoptysis: comparative study of the role of CT and fibreoptic bronchoscopy. Radiology 1993;189:677-80.

16 Naidich DP, Funt S, Ettenger NA, et al. Haemoptysis: CT-bronchoscopic correlations in 58 cases. Radiology 1990;177:357-62.

17 Hirshberg B, Biran I, Glazer M, et al. Haemoptysis: aetiology, evaluation, and outcome in a tertiary referral hospital. Chest 1997;112:440-4. 